\title{
A Scoping Review of Combined Yoga and Resistance Exercise for Dyspnea in Lung Cancer Survivors
}

\author{
Shreya Rewar ${ }^{1}$, Mona Al Onazi ${ }^{1}$, Kaitlyn Boudreau ${ }^{1}$ and Margaret L McNeely ${ }^{1,2 *}$ \\ ${ }^{1}$ Department of Physical Therapy, University of Alberta, Canada \\ ${ }^{2}$ Rehabilitation Medicine, Cross Cancer Institute, Canada
}

Submission: February 12, 2018; Published: June 22, 2018

*Corresponding author: Margaret L McNeely, Department of Physical Therapy, University of Alberta, 2-50 Corbett Hall, Edmonton, Alberta T6G 2G4, Canada, Tel: +1-780-248-1531; Fax: +1-780-492-4429; Email: mmcneely@ualberta.ca

\begin{abstract}
Introduction: Lung cancer is the second most common cancer and is the leading cause of death from cancer. Dyspnea, a self-reported subjective feeling of shortness of breath or breathlessness, is a common symptom experienced by survivors, especially those with advanced stage lung cancer.

Objectives: The purpose of this paper was to (1) perform a scoping review of the literature on yoga and resistance exercise interventions that included a breathing or pulmonary rehabilitation component to address dyspnea in survivors with lung cancer and (2) propose a physiotherapeutic protocol combining yoga with resistance exercise.

Results: A total of 3 single-group studies were found that examined supervised yoga interventions for survivors with lung cancer and 5 RCTs were found examining resistance exercise including a pulmonary rehabilitation component. The three yoga studies involved a total of 28 survivors with non-small cell lung cancer. Findings support feasibility and preliminary efficacy for sleep, mood, anxiety and aspects of quality of life. Five studies, involving 257 survivors with both small cell and non-small cell lung cancer, were found that examined combined resistance exercise intervention and a pulmonary rehabilitation component. Three studies were prehabilitation interventions carried out prior to lung cancer surgery. Benefits were found for measures of lung capacity, six-minute-walk-test distance and quality of life. Based on the findings, a physiotherapeutic protocol is proposed.
\end{abstract}

Conclusion: Given the scope of practice of physiotherapists and their training in cardiorespiratory therapy, it is hoped that this paper will encourage collaboration with yoga professionals to lead future research in the area.

Keywords: Exercise; Yoga; Physical therapy; Scoping review; Respiratory muscle retraining; Dyspnea

Abbreviations: 6MWT: Six-Minute Walk Test; RCT: Randomized Controlled Trial; SCLC: Small Cell Lung Cancer; NSCLC: Non Small Cell Lung Cancer

\section{Introduction}

Fifty percent of newly diagnosed cancers include those of lung and bronchus, breast, colorectal and prostate cancer [1]. Lung cancer is the second most common cancer diagnosed among males and females each accounting for $14 \%$ of all new cases and is the leading cause of death from cancer [1]. The diagnosis of lung cancer leads to debilitating symptoms for the survivor, not only from the cancer itself but also from the side effects of the treatment [2].

The largest proportion of cancer cases occur in older adults, and lung cancer is the most common cancer for individuals $70+$ years of age for both genders [1]. Unfortunately, older age is associated with increased symptom burden from the disease and its treatment [3]. Authors of a recent qualitative study reported adverse effects including pain, fatigue, weakness and dyspnea [2]. Dyspnea, a self-reported subjective feeling of shortness of breath, or breathlessness, is a common symptom experienced by survivors, especially those with advanced stage lung cancer
$[2,4,5]$. Although highly prevalent and distressing, the impact of dyspnea is often under-recognized despite its negative effects on physical and psychological functioning [5].

Exercise has shown benefit in addressing pain, fatigue, physical fitness and quality of life across cancer types [6,7]. Exercise may prove beneficial for those with lung cancer by increasing muscle strength, attenuating losses in muscle mass, reducing fatigue, and by helping survivors cope with physical and emotional adverse effects related to the disease and its treatment [8]. Survivors, however, report numerous barriers to exercise including low motivation; fear to exercise; lack of knowledge about benefits; and external barriers related to the environment, social support, and symptoms [9]. In particular, the fear of worsening dyspnea with movement and physical activity is a primary reason for survivor unwillingness to participate in exercise $[8,10]$. Thus, there is a need for further exploration of personalized exercise interventions for survivors of lung cancer 
that address overall fitness and functioning, while considering symptoms such as dyspnea.

Resistance exercise training may prove valuable to attenuate both sarcopenia and disease related declines in muscle mass commonly seen in survivors with lung cancer [11]. In addition to improving muscle mass, resistance exercise has been shown to increase peak oxygen uptake in deconditioned individuals and muscle strength in older adults [12]. Importantly, resistance exercise training has been shown to improve an individual's ability to carry out daily activities, and to reduce symptoms of fatigue in both healthy and chronic disease populations $[13,14]$. Yoga is now widely practiced throughout the world as a mind-body therapy and is considered part of complementary and alternative medicine. Given its gentle nature and focus on breathing and meditation, yoga may address barriers related to dyspnea, and thus prove a viable exercise option for survivors of lung cancer [10].

The purpose of this paper was to review the potential of a physiotherapeutic approach to address dyspnea in survivors with lung cancer that involves combining yoga with resistance exercise training. First, we provide a summary of the evidence on the benefits of exercise interventions in survivors with lung cancer by highlighting findings of key systematic reviews in the area. Next, we present the findings of our scoping review on yoga and resistance exercise interventions for dyspnea in lung cancer. Noting the limited direct research in the area of combined yoga and resistance exercise interventions, we propose a protocol for a physiotherapeutic approach involving yoga and resistance exercise for survivors of lung cancer with a focus on dyspnea.

\section{Exercise in Lung Cancer}

A Cochrane Systematic Review examined exercise interventions following lung cancer resection [15]. Three randomized controlled trials (RCTs), with 178 patients, were included in the review. Studies examined combinations of aerobic and resistance exercise training, with only one study including a focus on breathing/dyspnea management. Findings showed a statistically and clinically significant benefit for sixminute walk test (6MWT) distance $(50.4 \mathrm{~m} ; 95 \%$ confidence interval (CI): 15.4, 85.2). No significant benefits were found for quality of life or measures of lung function.

Another recent systematic review examined the benefits of home-based prehabilitation and rehabilitation programs for survivors with non-small cell lung cancer [16]. The review included 11 intervention studies comprising home-based or combined home and clinic/hospital-based supervised exercise. While benefits were shown for physical fitness outcomes, most of the studies involved survivors with early stage lung cancer and only two studies included a focus on breathing/dyspnea management. Importantly, low adherence rates to exercise were common, with studies involving regular supervision and personalized exercise resulting in better adherence and retention of participants [16].

Exercise training has also shown promise for managing dyspnea, both as a prehabilitation intervention and also when delivered as an intervention in the early post-surgical time period for lung cancer [12]. In a systematic review, including 15 studies, interventions primarily involved aerobic (walking and cycling) and breathing exercises. Eight studies involved prehabilitation exercise training ( $\mathrm{n}=8$ studies) and findings showed shorter lengths of hospital stays, decreased postoperative complications, and increased 6MWT distance. Seven studies involved postoperative exercise interventions and were found to improve both the 6MWT distance and dyspnea score in survivors [12].

\section{Scoping review on Yoga and Resistance Exercise for Dyspnea}

\section{Methods}

A scoping literature search of various databases including Medline, CINAHL, Embase and PEDRO was performed to find articles related to lung cancer and combined yoga and resistance exercise with a focus on breathing/dyspnea. As no studies were found examining the combination of interventions, we turned our attention to articles involving (1) yoga with an emphasis on breathing/ dyspnea and (2) resistance exercise intervention that included a breathing or pulmonary rehabilitation component.

Participants: Participants were required to be adults (17 years and older), diagnosed with lung cancer, where the intervention was in an outpatient hospital or in a communitybased setting. Participants could be actively receiving cancer treatment or be in the post-treatment phase at the time of the intervention.

Intervention: The primary intervention was supervised yoga with a breathing or meditation component OR supervised resistance exercise intervention with a breathing or pulmonary rehabilitation component. Programs that comprised homebased interventions alone were excluded.

Outcomes: Studies were required to include one of the following outcomes: dyspnea or a measure of lung function, fatigue, muscle strength and quality of life.

Study design: As we anticipated fewer trials in the yoga area, we considered clinical trials including single group pre-post designs, controlled trials and randomized controlled trials. Only randomized controlled trials were included for the resistance exercise studies. Studies were required to be published in English.

Scoping review procedures: Four review members screened the articles for inclusion in the review (SR, MAO, $\mathrm{KB}, \mathrm{MM}$ ). Studies meeting the eligibility criteria underwent independent data abstraction and review by three members of 
the review team (SR, MAO, KB). Information regarding study population, tumour group, methods, interventions, outcomes and adverse events were collected using a structured data abstraction form. Discrepancies were settled by consensus and if necessary, involved a fourth member of the review team (MM).

\section{Results}

A total of 3 studies $[10,17,18]$ were found that examined supervised yoga interventions for survivors with lung cancer and a total of 5 RCTs [19-23] were found examining resistance exercise including a pulmonary rehabilitation component. The three yoga studies used a single-group design and involved a total of 28 survivors with non-small cell lung cancer. Interventions involved Hatha $(n=2)$ or Tsa Lung yoga $(n=1)$, and all involved a breathing component. One study included both survivors and their caregivers. Findings support feasibility and preliminary efficacy for sleep, mood, anxiety and aspects of quality of life. No studies reported outcomes related to dyspnea or a measure of lung function. Further details on the included studies are provided in Table 1.

Table 1: Yoga studies with breathing component.

\begin{tabular}{|c|c|c|c|c|c|c|}
\hline $\begin{array}{l}\text { Author/Year/ } \\
\text { Country }\end{array}$ & $\begin{array}{c}\text { Study } \\
\text { Design/ } \\
\text { Sample } \\
\text { size (N) }\end{array}$ & $\begin{array}{l}\text { Patient } \\
\text { Details }\end{array}$ & $\begin{array}{l}\text { Intervention } \\
\text { Details }\end{array}$ & $\begin{array}{l}\text { Outcome } \\
\text { Measures }\end{array}$ & Study Results & Key Features \\
\hline $\begin{array}{c}\text { Fouladbakhsh/2014 } \\
\text { [10] United States }\end{array}$ & $\begin{array}{l}\text { One group, } \\
\text { repeated } \\
\text { measured design } \\
\text { Sample size: } \\
\text { N=9 }\end{array}$ & $\begin{array}{c}\text { NSCLC } \\
\text { Stages I-IIIa Post } \\
\text { initial } \\
\text { cancer } \\
\text { treatment }\end{array}$ & $\begin{array}{c}\text { Hatha Yoga: } \\
\text { Viniyoga } \\
\text { method } \\
\text { 1x40mins/week } \\
\text { for } 14 \text { weeks } \\
\text { 3-week pre- } \\
\text { intervention, } \\
\text { 8-week } \\
\text { yoga } \\
\text { intervention, } \\
\text { 3-week post } \\
\text { intervention } \\
\text { Follow up: } 3 \text { \& } 6 \\
\text { months }\end{array}$ & $\begin{array}{c}\text { Sleep quality } \\
\text { QoL Stress } \\
\text { (salivary cortisol) }\end{array}$ & $\begin{array}{c}\text { Significant } \\
\text { improvement } \\
\text { in sleep efficiency } \\
\text { overtime }(\mathrm{p}<0.02) \text {, } \\
\text { mood }(\mathrm{p}<0.02) \\
\text { and } \\
\text { quality of life } \\
\text { (QOL) } \\
\text { mental \& physical } \\
\text { subscales } \\
\text { (p<0.014), } \\
\text { increase in } \\
\text { physical health } \\
\text { scores }(\mathrm{p}<0.0001)\end{array}$ & $\begin{array}{l}\text { Modification } \\
\text { of poses for } \\
\text { individual } \\
\text { needs, Patient } \\
\text { education, Focus } \\
\text { on meditation, } \\
\text { postures, and } \\
\text { breathing } \\
\text { exercises to } \\
\text { deepen and slow } \\
\text { the breath }\end{array}$ \\
\hline $\begin{array}{c}\text { Milbury/2015 [17] } \\
\text { United States }\end{array}$ & $\begin{array}{l}\text { Pilot couple based } \\
\text { yoga program } \\
\text { Sample } \\
\text { size: Patient } \\
(n=10) ; \text { Caregiver } \\
(n=10)\end{array}$ & $\begin{array}{l}\text { NSCLC Stages } \\
\text { I-IV } \\
\text { Receiving at } \\
\text { least } 5 \text { weeks of } \\
\text { radiation } \\
\text { therapy }\end{array}$ & $\begin{array}{l}\text { Couple-based } \\
\text { Tsa Lung } \\
\text { yoga } 2-3 \times 45- \\
60 \mathrm{~min} / \text { week } \\
\text { over course of } \\
5-6 \text { week }\end{array}$ & $\begin{array}{l}\text { QoL Psychological } \\
\text { distress Sleep } \\
\text { disturbances } \\
\text { Fatigue } \\
\text { Health related } \\
\text { QoL } \\
\text { Spiritual well- } \\
\text { being }\end{array}$ & $\begin{array}{c}\text { Feasibility: } 80 \% \\
\text { attended } \\
\text { at least } 50 \% \text { of } \\
\text { sessions } \\
\text { Significant: } \\
\text { Increase in } \\
\text { spiritual well- } \\
\text { being (p=0.03) } \\
\text { Medium effect: } \\
\text { Sleep } \\
\text { disturbance } \\
\text { (d=0.60), } \\
\text { decreased } \\
\text { depressive } \\
\text { symptoms } \\
\text { (d=0.52) }\end{array}$ & $\begin{array}{c}\text { Dyadic } \\
\text { intervention and } \\
\text { analysis } \\
\text { Program well } \\
\text { accepted } \\
\text { with high rates of } \\
\text { class \& } \\
\text { at home practice } \\
\text { Caregiver results: } \\
\text { significant } \\
\text { decrease in } \\
\text { fatigue, and } \\
\text { anxiety }\end{array}$ \\
\hline $\begin{array}{c}\text { Milbury/2015 [18] } \\
\text { United States }\end{array}$ & $\begin{array}{l}\text { Study Design: } \\
\text { Single- arm } \\
\text { feasibility study } \\
\text { Sample size: } \\
(\mathrm{N}=9)\end{array}$ & $\begin{array}{l}\text { NSCLC Stages } \\
\text { I-IIIB Receiving } \\
\text { at least } 5 \text { weeks } \\
\text { of radiotherapy }\end{array}$ & $\begin{array}{l}\text { Hatha Yoga } \\
\text { (Vivekananda } \\
\text { Method: couple- } \\
\text { based) } 2-3 \\
\text { sessions for } \\
\text { total of } 60 \mathrm{mins} \text { / } \\
\text { week } 15 \text { sessions } \\
\text { over course of } \\
\text { 5-6 weeks of } \\
\text { radiotherapy }\end{array}$ & $\begin{array}{l}\text { QoL, } \\
\text { Psychological, } \\
\text { distress, Well- } \\
\text { being, Fatigue }\end{array}$ & $\begin{array}{c}\text { Significant: } \\
\text { Decrease in } \\
\text { anxiety }(\mathrm{p}=0.04) \text {, } \\
\text { increase } \\
\text { in mental health } \\
\text { aspects of } \\
\text { QoL }(\mathrm{P}=0.04) \\
\text { Medium } / \text { Small } \\
\text { effect: } \\
\text { Sleep disturbance } \\
\text { (d=0.65) } \\
\text { and spiritual well- } \\
\text { being }(\mathrm{d}=0.64) \text {, } \\
\text { and somatization }\end{array}$ & $\begin{array}{l}\text { Couples-based } \\
\text { program (dyadic } \\
\text { approach) } \\
\text { Feasible, } \\
\text { acceptable, } \\
\text { and safe for the } \\
\text { lung } \\
\text { cancer } \\
\text { population } \\
\text { Caregiver results: }\end{array}$ \\
\hline
\end{tabular}




\begin{tabular}{|l|l|l|l|l|c|}
\hline & & & & & \\
& & & & & $\begin{array}{c}\text { Significant: Sleep } \\
\text { disturbances } \\
\text { p-values given } \\
\text { Medium effect: } \\
\text { improved } \\
\text { physical aspects } \\
\text { of QoL }\end{array}$ \\
\hline
\end{tabular}

NSCLC: Non-Small Cell Lung Cancer; QoL: Quality of Life; EORTC QLQ: European Organisation for Research and Treatment of Cancer Quality of Life Questionnaire; $d=$ effect size

Five studies [19-23], involving 257 survivors with both small cell and non-small cell lung cancer, were found that examined resistance exercise along with a pulmonary rehabilitation component (Table 2). One study was carried out in the posttreatment phase and involved a 10-week group-based supervised exercise program, once a week. No significant differences were found between the intervention and control group for any outcomes. Three studies were prehabilitation interventions carried out prior to lung cancer surgery. Benefits were found for measures of lung capacity, 6MWT distance and quality of life. The final study examined exercise during palliative chemotherapy and showed benefit for daily activities, functional capacity and symptoms of dyspnea.

Table 2: Resistance exercise studies with breathing or respiratory muscle retraining.

\begin{tabular}{|c|c|c|c|c|c|c|}
\hline $\begin{array}{l}\text { Author/ } \\
\text { Year/ } \\
\text { Country }\end{array}$ & $\begin{array}{c}\text { Study Design \& } \\
\text { Sample size }\end{array}$ & $\begin{array}{c}\text { Patient } \\
\text { Characteristics }\end{array}$ & $\begin{array}{l}\text { Intervention } \\
\text { Details }\end{array}$ & $\begin{array}{l}\text { Outcome } \\
\text { Measures }\end{array}$ & Study Results & Key Features \\
\hline $\begin{array}{l}\text { Huang } 2017 \text { [19] } \\
\text { China }\end{array}$ & $\begin{array}{c}\text { Three-arm RCT } \\
\text { Sample size: } \\
\text { N=90 }\end{array}$ & $\begin{array}{c}\text { NSCLC, Pre- } \\
\text { lobectomy } \\
\text { Age (mean } \pm \text { SD): } \\
63.6 \pm 6.8 \\
\text { Stage I-III, COPD } \\
\text { with a } \\
\text { heavy smoking } \\
\text { history }\end{array}$ & $\begin{array}{l}\text { 1) Combined Pre- } \\
\text { operative } \\
\text { Pulmonary } \\
\text { Rehabilitation (PR) } \\
\text { group: one week } \\
\text { with high- } \\
\text { intensity } \\
\text { preoperative PR } \\
\text { (inspiratory muscle } \\
\text { training } \\
\text { (IMT)+resistance } \\
\text { exercise } \\
\text { 2) IMT-alone group: } \\
\text { conventional } \\
\text { single-mode IMT } \\
\text { 3) control group: } \\
\text { routine } \\
\text { preoperative care }\end{array}$ & $\begin{array}{l}\text { 6-MWD, Peak, } \\
\text { expiratory flow } \\
\text { (PEF), Fatigue, } \\
\text { Dyspnea index, } \\
\text { QoL (EORTC- } \\
\text { QLQ-C30 and } \\
\text { EORTC-LC13) }\end{array}$ & $\begin{array}{c} \\
\text { PR vs Control: } \\
\text { 6-MWD }(\mathrm{P}=0.002) \\
\text { and PEF }(\mathrm{P}=0.001) \text {, } \\
\text { QOL scores: } \\
\text { significant } \\
\text { difference }(\mathrm{P}=0.035) \\
\text { Global } \\
\text { QoL in favour of PR, } \\
\text { PR vs IMT group: } \\
\text { PEF (P=0.004) in } \\
\text { favour of PR }\end{array}$ & $\begin{array}{l}\text { Short term, high } \\
\text { intensity } \\
\text { pulmonary } \\
\text { prehabilitation } \\
\text { program } \\
\text { including } \\
\text { IMT and } \\
\text { resistance } \\
\text { exercise. }\end{array}$ \\
\hline $\begin{array}{c}\text { Henke/2014 [20] } \\
\text { Germany }\end{array}$ & $\begin{array}{l}\text { RCT Sample size: } \\
\text { N=46 } \\
\text { (29 patients } \\
\text { completed the } \\
\text { trial) }\end{array}$ & $\begin{array}{l}\text { NSCLC and SCLC, } \\
\text { stages, IIIA/ } \\
\text { IIIB/IV, receiving } \\
\text { inpatient } \\
\text { palliative } \\
\text { platinum-based } \\
\text { chemotherapy }\end{array}$ & $\begin{array}{c}\text { Intervention } \\
\text { group (IG): } \\
\text { Endurance training } \\
\text { (walking exercise) } \\
\text { and breathing } \\
\text { techniques, } \\
\text { (5 days/ } \\
\text { week)+resistance } \\
\text { training (every } \\
\text { other day) } \\
\text { Control group } \\
\text { (CG):Conventional } \\
\text { physiotherapy }\end{array}$ & $\begin{array}{l}\text { Barthel Index } \\
\text { 6MWD and } \\
\text { staircase } \\
\text { walking } \\
\text { Dyspnea: } \\
\text { Modified Borg } \\
\text { Scale (MBS), } \\
\text { QoL: (EORTC } \\
\text { QLQ-C30/LC13) }\end{array}$ & $\begin{array}{c}\text { Barthel Index } \\
\text { (p=0.041) in } \\
\text { favour of IG } \\
\text { Functional capacity: } \\
\text { 6MWD, staircase } \\
\text { walking } \\
\text { exercise, and } \\
\text { strength } \\
\text { capacity in favour } \\
\text { of IG } \\
\text { (p<0.05) } \\
\text { Dyspnea: Significant } \\
\text { decrease in the } \\
\text { level of } \\
\text { dyspnea in the IG, } \\
\text { (p<0.05) } \\
\text { QoL: Significant } \\
\text { differences } \\
\text { in specific } \\
\text { component scores } \\
\text { only }\end{array}$ & $\begin{array}{c}\text { Benefit of } \\
\text { enhanced } \\
\text { physiotherapy } \\
\text { including } \\
\text { endurance } \\
\text { training and } \\
\text { strength training } \\
*\end{array}$ \\
\hline
\end{tabular}




\begin{tabular}{|c|c|c|c|c|c|c|}
\hline $\begin{array}{l}\text { Brocki/ } 2014 \text { [21] } \\
\text { Ireland }\end{array}$ & $\begin{array}{l}\text { RCT Sample size: } \\
\qquad \mathrm{N}=78\end{array}$ & $\begin{array}{c}\text { Age (mean } \pm \text { SD): } \\
64.5 \pm 9.5, \text { Radical } \\
\text { Surgery } \\
\text { for lung cancer }\end{array}$ & $\begin{array}{c}\text { 10-week group- } \\
\text { based supervised } \\
\text { exercise } \\
\text { programme, once a } \\
\text { week: } 15 \mathrm{~min} \\
\text { warming up, } \\
\text { followed by } 20 \mathrm{~min} \\
\text { aerobic } \\
\text { exercise, } 15 \mathrm{~min} \\
\text { muscle strength } \\
\text { training and } 10 \mathrm{~min} \\
\text { cooling } \\
\text { down/relaxation, } \\
\text { Intervention } \\
\text { included dyspnea } \\
\text { management } \\
\text { techniques. }\end{array}$ & $\begin{array}{l}\text { Health-related } \\
\text { QoL: 36- } \\
\text { Item Short } \\
\text { Form Health } \\
\text { Survey version } \\
2 \text { (SF36), } \\
\text { Functional } \\
\text { exercise } \\
\text { capacity: } \\
\text { 6MWT, Lung } \\
\text { function: } \\
\text { spirometry }\end{array}$ & $\begin{array}{c}\text { No difference } \\
\text { between } \\
\text { groups at any time- } \\
\text { point. } \\
\text { Both groups } \\
\text { increased their } \\
\text { walking distance (IG: } \\
61 \mathrm{~m} \text {, } \\
\text { 95\% CI: [43;79] and } \\
\text { CG: } 55 \\
\text { m, 95\% CI: [40;70]) } \\
\text { and this } \\
\text { increase was } \\
\text { sustained after } \\
\text { one year }\end{array}$ & $\begin{array}{l}\text { The exercise } \\
\text { programme was } \\
\text { personalized } \\
\text { according to } \\
\text { physical } \\
\text { capability and } \\
\text { submaximal } \\
\text { exercise } \\
\text { test. Home } \\
\text { exercises } \\
\text { given to both } \\
\text { groups }\end{array}$ \\
\hline $\begin{array}{c}\text { Morano/2014 } \\
\text { [22] Brazil }\end{array}$ & $\begin{array}{l}\text { Study design: } \\
\text { Randomized } \\
\text { single-blinded } \\
\text { exploratory } \\
\text { studies } \\
\text { Sample size: } \\
\text { N=19; Control } \\
\text { n=9; PR n=10 }\end{array}$ & $\begin{array}{c}\text { Patients } \\
\text { undergoing } \\
\text { lung cancer } \\
\text { resection and with } \\
\text { moderate-severe } \\
\text { COPD }\end{array}$ & $\begin{array}{l}10 \text { face to face } \\
\text { session in one } \\
\text { week (twice a day) } \\
\text { Preoperative } \\
\text { pulmonary } \\
\text { rehabilitation: } \\
\text { 1. Endurance } \\
\text { training } 20 \text { min } \\
\text { target: treadmill or } \\
\text { Nu-step or } \\
\text { arm ergometer or } \\
\text { arm-R-size } \\
\text { exercise, } \\
2 . \\
\text { Strengthening(UE/ } \\
\text { LE } \\
\text { alternating every } \\
\text { other day) } \\
\text { using theraband: } 2 \\
\text { sets of } 10-12 ; \\
\text { 3. Inspiratory } \\
\text { muscle training } \\
\text { IMT: 15-20 min of } \\
\text { daily use } \\
\text { Slow breathing: } 10 \\
\text { min each } \\
\text { session, prolonged } \\
\text { expiratory } \\
\text { time using pursed } \\
\text { lips Weekend } \\
\text { set collaboratively }\end{array}$ & $\begin{array}{c}\text { Postoperative } \\
\text { pulmonary } \\
\text { complications } \\
\text { and mechanical } \\
\text { ventilation } \\
\text { (they measured } \\
\text { only } \\
\text { baseline values } \\
\text { of pulmonary } \\
\text { function and } \\
\text { dyspnea } \\
\text { scores) }\end{array}$ & $\begin{array}{l}\text { Short term } \\
\text { preoperative } \\
\text { pulmonary } \\
\text { rehabilitation is } \\
\text { feasible. No } \\
\text { statistically } \\
\text { significant findings. }\end{array}$ & $\begin{array}{c}\text { Finding of } \\
\text { shorter time of } \\
\text { chest tube may } \\
\text { indicate a better } \\
\text { lung re- } \\
\text { expansion, a } \\
\text { results that may } \\
\text { be associated } \\
\text { with routine use } \\
\text { of IMT }\end{array}$ \\
\hline $\begin{array}{c}\text { Morano/2013 } \\
\text { [23] Brazil }\end{array}$ & $\begin{array}{l}\text { Randomized } \\
\text { clinical trial } \\
\text { Sample Size: } \mathrm{n}=24 \\
\text { Pulmonary } \\
\text { rehabilitation } \\
\text { (PR) group: } \\
\text { n=12(recruited) } \\
\text { n=12 completed } \\
\text { Chest } \\
\text { physiotherapy }\end{array}$ & $\begin{array}{c}\text { Patients } \\
\text { undergoing lung } \\
\text { cancer resection } \\
\text { for NSCLC } \\
\text { Age: } \\
\text { CPT: } 68.8 \pm 7.3 \\
\text { PR: } 64.8 \pm 8\end{array}$ & $\begin{array}{c}4 \text { weeks of } \\
\text { preoperative } \\
\text { pulmonary } \\
\text { rehabilitation (PR) } \\
\text { VS } \\
\text { CPT } \\
\text { PR: } 1 \text {. UE PNF } \\
\text { pattern with light } \\
\text { weight } 2 \text {. LE } \\
\text { endurance on }\end{array}$ & $\begin{array}{l}\text { Spirometry: } \\
\text { Fev1, FVC, } \\
\text { MIP(maximal } \\
\text { inspiratory } \\
\text { pressure), } \\
\text { MEP(maximal } \\
\text { expiratory } \\
\text { pressure) } \\
\text { Quality of life: } \\
\text { SF-36 }\end{array}$ & $\begin{array}{c}\text { Significant increase: } \\
\text { FVC in Litres and \% } \\
(\mathrm{p}=.02, \\
\mathrm{p}=.00 \text { respectively) } \\
\text { MIP }(\mathrm{P}=.00) \\
\text { MEP }(\mathrm{P}=.00) \text { SF-36 } \\
\text { physical component } \\
\text { summary }(\mathrm{p}=0.07)\end{array}$ & $\begin{array}{l}\text { Improvement in } \\
\text { preoperative } \\
\text { functional } \\
\text { capacity, fewer } \\
\text { postoperative } \\
\text { pulmonary } \\
\text { complications, } \\
\text { postoperative } \\
\text { stay. }\end{array}$ \\
\hline
\end{tabular}




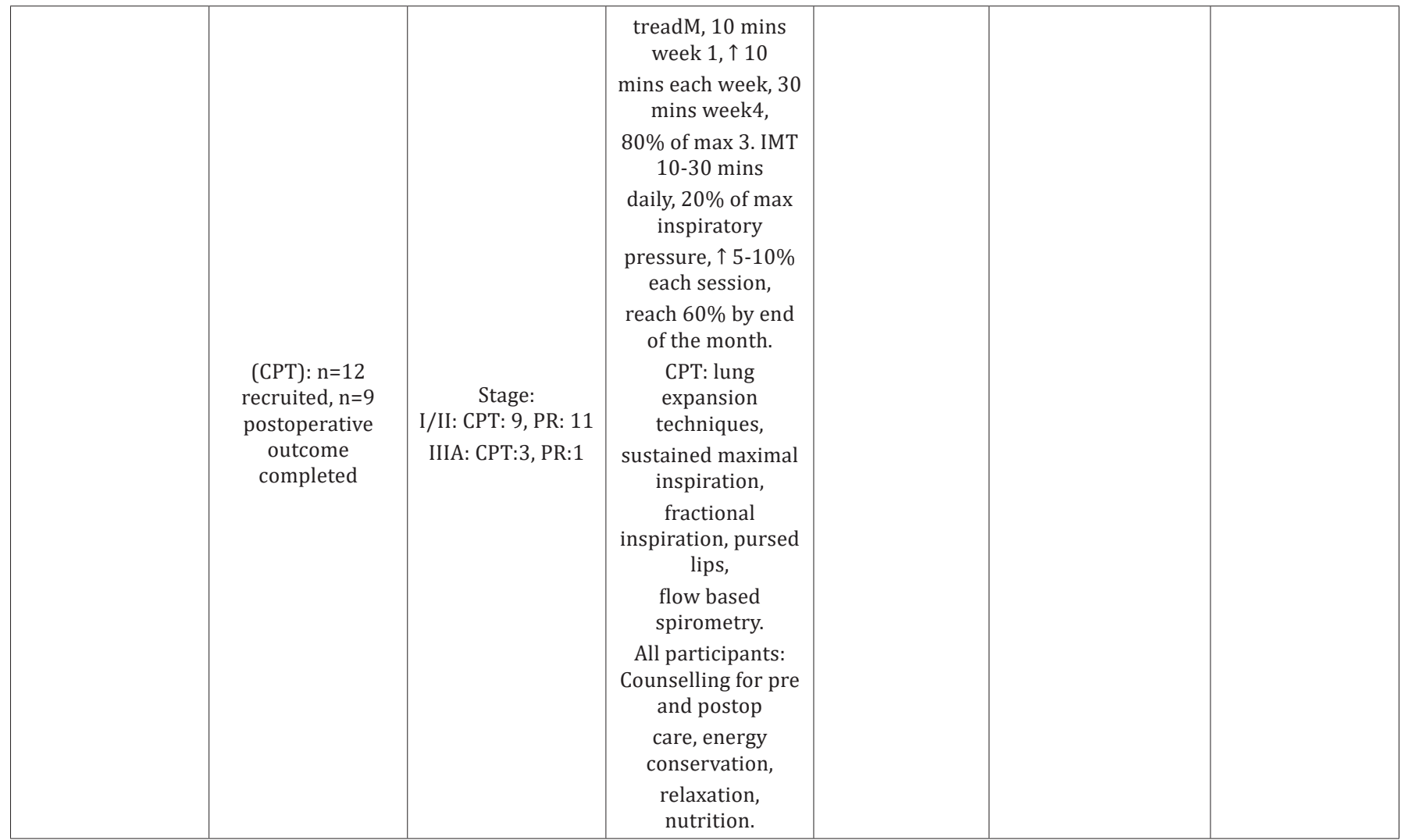

SCLC: Non-Small Cell Lung Cancer; QoL: Quality of Life; EORTC QLQ: European Organisation for Research and Treatment of Cancer Quality of Life Questionnaire; SF-36: medical Outcomes Short Form 36 Quality of Life Questionnaire.

\section{Discussion}

\section{Proposed physiotherapeutic yoga protocol}

Current exercise guidelines for cancer largely reflect physical activity recommendations for the general population [24]. At present, the most beneficial exercise regimen for survivors of lung cancer in terms of type, frequency, and duration is currently not known $[12,15,16,25,26]$. Based on current evidence, it is likely that an exercise program including a therapeutic yoga focus on the mechanics of breathing as well as a resistance exercise program that includes inspiratory muscle training would be both acceptable to, and beneficial for survivors (Figure 1). Table 3 includes a rationale for a combined physiotherapeutic yoga and resistance exercise protocol that aims to address the needs of survivors of lung cancer. Key components of the combined intervention include yoga practice with attention to the mechanics of breathing, resistance exercise including inspiratory muscle retraining, and a cool-down with a focus on stretching of key muscles of respiration.

Table 3: Proposed protocol for physiotherapeutic and yoga exercise program.

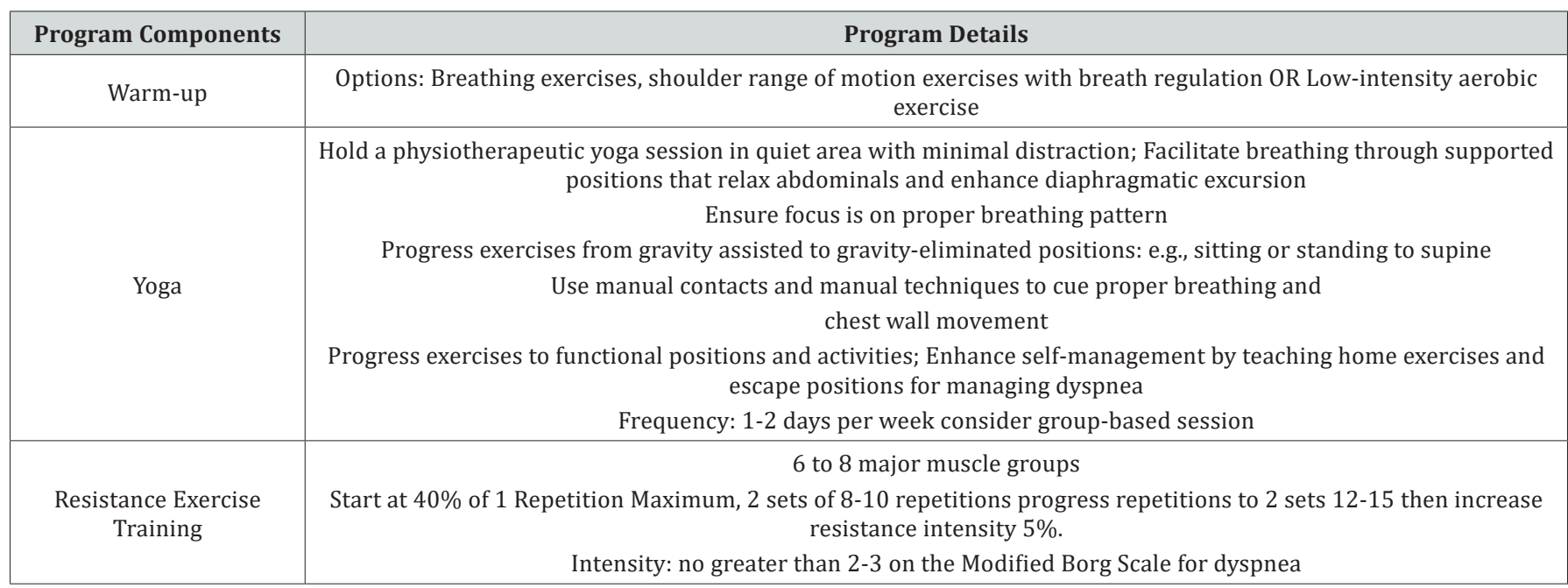


Journal of Yoga and Physiotherapy

\begin{tabular}{|c|c|}
\hline & $\begin{array}{c}\text { Frequency: minimum 2 days per week } \\
\text { Consider Inspiratory Muscle Retraining with respiratory training device: 10 minutes progressing to 30 minutes daily }\end{array}$ \\
\hline $\begin{array}{c}\text { Cool-down: focus on } \\
\text { stretching of inspiratory } \\
\text { \& expiratory muscles }\end{array}$ & $\begin{array}{c}\text { Scalenes, pectoralis major \& minor, latissimus dorsi, serratus anterior, } \\
\text { rectus abdominus and internal \& external obliques }\end{array}$ \\
\hline $\begin{array}{c}\text { Reduce workload/ } \\
\text { discontinue exercise }\end{array}$ & $\begin{array}{c}\text { Excessive fatigue post-exercise } \\
\text { Muscle soreness }>48 \text { hours }\end{array}$ \\
& $\begin{array}{c}\text { Exacerbation of dyspnea ( }>3), \text { excessive coughing or increase in pain } \\
\text { during or following sessions }\end{array}$ \\
\hline
\end{tabular}

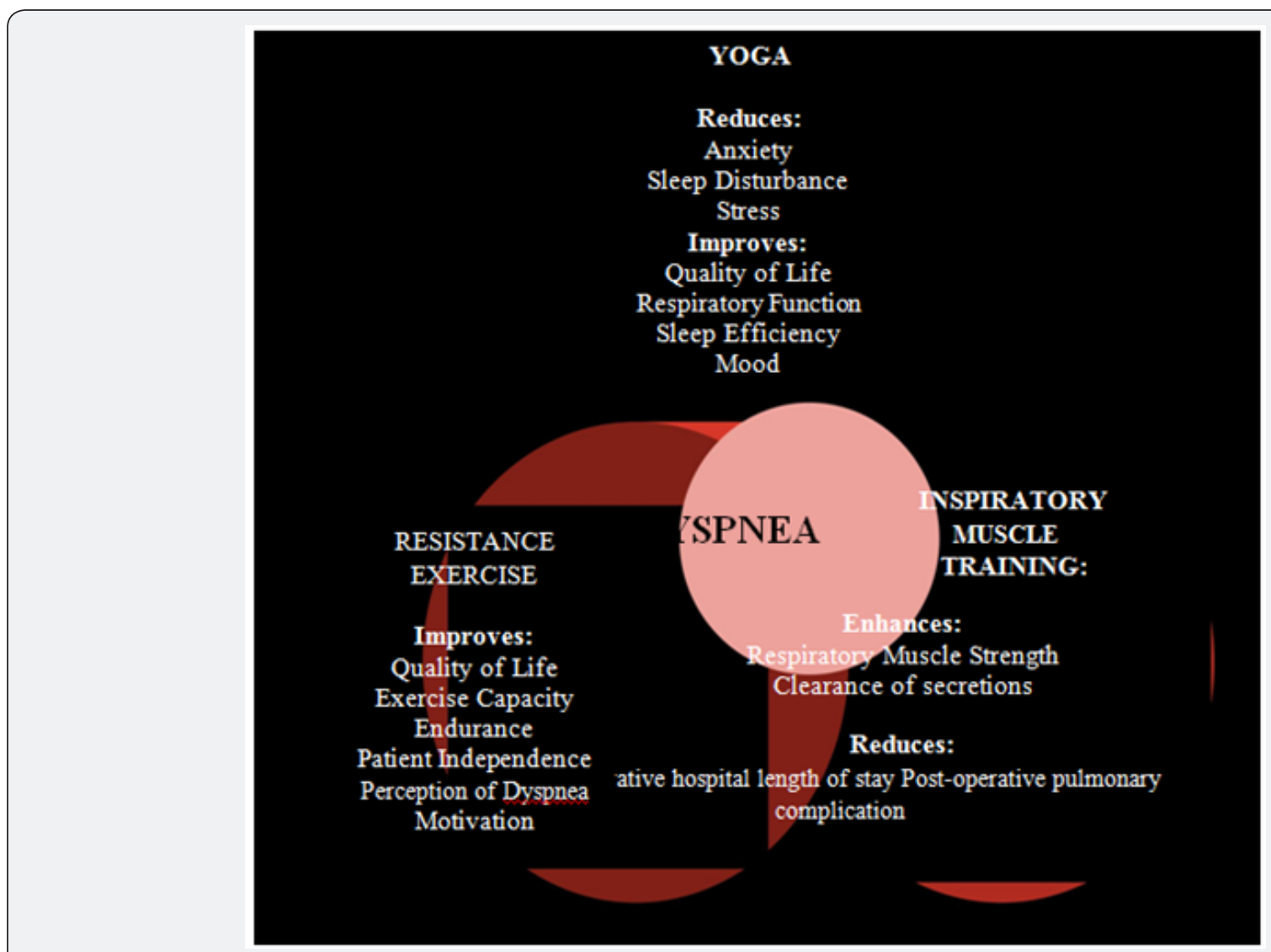

Figure 1: Potential role of exercise in addressing Dyspnea.

Resistance exercise: Resistance exercise training optimizes physical efficiency and performance $[27,28]$. With repeated bouts of appropriately prescribed resistance exercise the musculoskeletal system undergoes a progressive positive adaptation to the imposed stress, and the survivor's ability to resist physical fatigue is enhanced [27]. Moreover, using the similar principles, respiratory muscle training may be used to optimize lung function by targeting the strength of inspiratory muscles. Deeper, more efficient breathing allows more oxygen to enter the bloodstream with each breath while strengthening the breathing muscles. Interventions may include teaching diaphragmatic breathing, segmental and purse-lipped breathing; and inspiratory muscle training (IMT) using a breathing device $[29,30]$. In other disease conditions, IMT has been shown to improve inspiratory muscle function, decrease symptoms of dyspnea and allow patients to exercise more comfortably [31].
As seen in the results of this scoping review, early evidence supports its use in survivors of lung cancer.

Yoga component: Yoga is a way of life as based on the eastern traditions of India, Tibet and China. Yoga consists of three principal components as pranayama (breathing exercises), Meditation (dhyana), and asanas (postures) [32]. There are many different styles and types of yoga commonly practiced in the western world. Hatha yoga is a traditional form of yoga from India. Hatha yoga involves a series physical postures and breathing techniques, and is a method used to calm the body, mind and spirit in preparation for meditation. Hatha yoga includes the styles of Ashtanga, Iyengar, Anusara, Vivekanada, and Vinyasa. Tsa Lung, a Tibetan form of yoga, uses breath retention techniques with physical movements and visualizations to promote relaxation and healing, and to still 
the mind. Early evidence from this scoping review suggests potential benefit from yoga for symptoms of dyspnea. Given other reported benefits of yoga for sleep, cancer-related fatigue, psychosocial distress, and musculoskeletal symptoms [32]; further investigation of yoga as an intervention for survivors with lung cancer is warranted.

Exercise safety considerations: Prior to performing exercise testing or training, information must be collected on important diagnostic and treatment variables such as the survivor's type and stage of lung cancer, cancer treatments training. received or ongoing, and identify any acute or chronic adverse effects related to the cancer and/or cancer treatment [24]. Table 4 provides a list of precautions/potential contraindications to exercise that includes considerations specific to lung cancer. Given the older age of survivors with lung cancer further screening for co-morbid conditions is needed. Following a simple screening tool such as the Revised PAR-Q (Canadian Society for Exercise Physiology's web site http://www.csep.ca) may be useful to identify survivors of lung cancer who require further medical evaluation prior to taking part in exercise testing or

Table 4: Precautions/potential contraindications to exercise specific to lung cancer.

\begin{tabular}{|c|c|c|}
\hline Body System & Precautions/Potential & Comments \\
\hline Musculoskeletal & $\begin{array}{l}\text { Bone, back or joint pain of recent } \\
\text { origin } \\
\text { Unusual muscular weakness } \\
\text { Severe Cachexia } \\
\text { Unusual/extreme fatigue }\end{array}$ & $\begin{array}{c}\text { High risk of bone metastases if } \\
\text { presenting with bone pain or } \\
\text { onset of unusual muscle } \\
\text { weakness Cachexia and exhaustion may be seen } \\
\text { in advanced lung cancer and may limit exercise } \\
\text { tolerance }\end{array}$ \\
\hline Cardiovascular & $\begin{array}{c}\text { Chest pain } \\
\text { Resting pulse }>100 / \text { min or }<50 / \mathrm{min} \\
\text { Resting blood pressure }>160 \\
\text { mm Hg systolic or }<85 \mathrm{mmHg} \text { and }>110 \mathrm{mmHg} \text { Diastolic } \\
\text { or }<50 \mathrm{mmHg} \\
\text { Irregular pulse } \\
\text { Swelling of ankles }\end{array}$ & $\begin{array}{l}\text { Presenting factors indicate higher risk of cardiac } \\
\text { event with exercise }\end{array}$ \\
\hline Pulmonary & $\begin{array}{c}\text { Severe dyspnea: respiration rate }>14 \text { breaths / minute at } \\
\text { rest } \\
\text { Coughing, wheezing } \\
\text { Chest pain increased by deep } \\
\text { breath } \\
\text { Oxygen saturation }<90 \% \\
\text { Dyspnea }>4 \text { on Borg } 10 \text {-point }\end{array}$ & Inadequate ventilator capacity for exercise \\
\hline
\end{tabular}

Pre-exercise screening should include assessment of the survivor's vital signs (blood pressure, heart rate, oxygen saturation, respiration rate and dyspnea evaluation), as an indication of overall health status [28]. Prior to assessment, survivors should rest for a period of at least 10 minutes. A heart rate monitor can be provided to the survivor with lung cancer to wear while exercising so that heart rate response can be easily observed. Vital signs should be taken before, during and after exercise testing and training to ascertain safety of exercise. Survivors with abnormal readings should refrain from exercise until such time as normal readings are obtained, or if remaining abnormal should be referred to their oncologist or primary care physician for further medical evaluation [28]. A dyspnea visual analog scale, such as the modified Borg Scale, can be used to measure the perceived level of breathlessness before and after the intervention [33]. Oxygen saturation levels can be monitored during exercise and may inform the need for rest/recovery.

Special considerations for dyspnea during Exercise: Simple interventions to relieve breathing distress during exercise, such as performing exercise in a supported sitting position, may be introduced to allow for increased lung expansion. In survivors with lung congestion, chest physical therapy techniques may be incorporated to open airways prior to exercise [34]. Recovery or escape positions to ease breathing should be demonstrated to the survivor with lung cancer and their caregivers as a means to manage episodes of dyspnea during exercise or daily activities [34]. Importantly, survivors should be taught to use escape positions to increase ventilatory capacity when experiencing dyspnea during exercise testing and training sessions. As an example, one escape position involves leaning forward in a seated position and supporting the thorax by bracing the forearms against a chair or on the knees. Alternatively, the survivor can lean against a wall in a similarly supported standing position [34]. Pacing is another important aspect for those with dyspnea and is critical to ensure exercise performance falls within the limits of a survivor's ventilatory capacity. If a survivor becomes slightly short of breath, they are instructed to stop the exercise, attain an escape position, and use controlled purse-lip breathing (to increase end-expiratory pressure and improve oxygenation) until the symptoms subside [34]. 


\section{Conclusion}

As can be seen from the foregoing review of the literature on yoga and resistance exercise for survivors of lung cancer, the body of literature has been minimal and research supporting the efficacy of interventions is generally lacking. There is a need for further research examining the benefits of a lung cancer-specific program involving combined yoga and resistance exercise training for symptoms of dyspnea. Given the scope of practice of physiotherapists and their training in cardiorespiratory therapy, it is hoped that this paper will encourage collaboration with yoga practitioners to lead future research examining rehabilitation strategies for dyspnea in survivors of lung cancer.

\section{References}

1. Canadian Cancer Society's Advisory Committee on Cancer Statistics (2017) Canadian Cancer Statistics. Toronto, ON, Canada.

2. Molassiotis A, Lowe M, Blackhall F, Lorigan P (2011) A qualitative exploration of a respiratory distress symptom cluster in lung cancer: cough, breathlessness and fatigue. Lung Cancer 71(1): 94-102.

3. Jalal SI, Riggs HD, Melnyk A, Richards D, Agarwala A, et al. (2012) Updated survival and outcomes for older adults with inoperable stage III non-small-cell lung cancer treated with cisplatin, etoposide, and concurrent chest radiation with or without consolidation docetaxel: analysis of a phase III trial from the Hoosier Oncology Group (HOG) and US Oncology. Ann Oncol 23(7): 1730-1738.

4. Yennurajalingam S, Lu Z, Williams JL, Liu DD, Arthur JA, et al. (2017) Characteristics of patients with advanced lung cancer referred to a vrapid-access supportive care clinic. Palliat Support Care 15(2): 197204.

5. Tanaka K, Akechi T, Okuyama T, Nishiwaki Y, Uchitomi Y (2002) Factors correlated with dyspnea in advanced lung cancer patients: organic causes and what else? J Pain Symptom Manage 23(6): 490-500.

6. Speck RM, Courneya KS, Mâsse LC, Duval S, Schmitz KH (2010) An update of controlled physical activity trials in cancer survivors: a systematic review and meta-analysis. J Cancer Surviv 4(2): 87-100.

7. Rock CL, Doyle C, Demark WW, Meyerhardt J, Courneya KS, et al. (2012) Nutrition and physical activity guidelines for cancer survivors. CA: a cancer journal for clinicians 62(4): 243-274.

8. Michaels C (2016) The importance of exercise in lung cancer treatment. Transl Lung Cancer Res 5(3): 235-238.

9. Granger CL, Denehy L, Remedios L, Retica S, Phongpagdi P, et al. (2016) Barriers to translation of physical activity into the lung cancer model of care. A qualitative study of clinicians' perspectives. Ann Am Thorac Soc 13(12): 2215-2222.

10. Fouladbakhsh JM, Davis JE, Yarandi HN (2014) A pilot study of the feasibility and outcomes of yoga for lung cancer survivors. Oncol Nurs Forum 41(2): 162-174.

11. Collins J, Noble S, Chester J, Coles B, Byrne A (2014) The assessment and impact of sarcopenia in lung cancer: a systematic literature review. BMJ Open 4(1): e003697.

12. Ni HJ, Pudasaini B, Yuan XT, Li HF, Shi L, et al. (2017) Exercise training for patient's pre and post surgically treated for non-small cell lung cancer: A systematic review and meta-analysis. Integr Cancer Ther 16(1): 63-73.

13. Strasser B, Siebert U, Schobersberger W (2013) Effects of resistance training on respiratory function in patients with chronic obstructive pulmonary disease: a systematic review and meta-analysis. Sleep Breath 17(1): 217-226.

14. Hagerman FC, Walsh SJ, Staron RS, Hikida RS, Gilders RM, et al. (2000)
Effects of high-intensity resistance training on untrained older men. I. Strength, cardiovascular, and metabolic responses. J Gerontol A Biol Sci Med Sci 55(7): B336-B346.

15. Cavalheri V, Tahirah F, Nonoyama M, Jenkins S, Hill K (2014) Exercise training for people following lung resection for non-small cell lung cancer-a Cochrane systematic review. Cancer Treat Rev 40(4): 585594.

16. Driessen EJ, Peeters ME, Bongers BC, Maas HA, Bootsma GP, et al. (2017) Effects of prehabilitation and rehabilitation including a homebased component on physical fitness, adherence, treatment tolerance, and recovery in patients with non-small cell lung cancer: A systematic review. Crit Rev Oncol Hematol 114: 63-76.

17. Milbury K, Chaoul A, Engle R, Liao Z, Yang C, et al. (2015) Couple-based Tibetan yoga program for lung cancer patients and their caregivers. Psychooncology 24(1): 117-120.

18. Milbury K, Mallaiah S, Lopez G, Liao Z, Yang C, et al. (2015) Vivekananda yoga program for patients with advanced lung cancer and their family caregivers. Integr Cancer Ther 14(5): 446-451.

19. Huang J, Lai Y, Zhou X, Li S, Su J, et al. (2017) Short-term highintensity rehabilitation in radically treated lung cancer: A three-armed randomized controlled trial. J Thorac Dis 9(7): 1919-1929.

20. Henke CC, Cabri J, Fricke L, Pankow W, Kandilakis G, et al. (2014) Strength and endurance training in the treatment of lung cancer patients in stages IIIA/IIIB/IV. Support Care Cancer 22(1): 95-101.

21. Brocki BC, Andreasen J, Nielsen LR, Nekrasas V, Gorst RA, et al. (2014) Short and long-term effects of supervised versus unsupervised exercise training on health-related quality of life and functional outcomes following lung cancer surgery-a randomized controlled trial. Lung Cancer 83(1): 102-108.

22. Morano MT, Mesquita R, Da Silva GP, Araújo AS, Pinto JM, et al. (2014) Comparison of the effects of pulmonary rehabilitation with chest physical therapy on the levels of fibrinogen and albumin in patients with lung cancer awaiting lung resection: a randomized clinical trial. BMC Pulm Med 14: 121.

23. Morano MT, Araújo AS, Nascimento FB, da Silva GF, Mesquita R, et al. (2013) Preoperative pulmonary rehabilitation versus chest physical therapy in patients undergoing lung cancer resection: a pilot randomized controlled trial. Arch Phys Med Rehabil 94(1): 53-58.

24. Schmitz KH, Courneya KS, Matthews C, Demark WW, Galvão DA, et al. (2010) American college of sports medicine roundtable on exercise guidelines for cancer survivors. Med Sci Sports Exerc 42(7): 14091426.

25. Cavalheri V, Tahirah F, Nonoyama M, Jenkins S, Hill K (2013) Exercise training undertaken by people within 12 months of lung resection for non-small cell lung cancer. Cochrane Database Syst Rev (7): CD0 09955.

26. Crandall K, Tahirah F, Nonoyama M, Jenkins S, Hill K, et al. (2014) Exercise intervention for patients surgically treated for non-small cell lung cancer (NSCLC): a systematic review. Surg Oncol 23(1): 17-30.

27. McArdle WD (2006) Exercise physiology: Energy, nutrition, and human performance. Lippincott Williams \& Wilkins, Philadelphia, USA.

28. American College of Sports Medicine (2017) ACSM's Guidelines for Exercise Testing and Prescription. Wolters Kluwer Health, $\left(10^{\text {th }} \mathrm{edn}\right)$, Philadelphia, USA.

29. Peddle CJ, Jones LW, Eves ND, Reiman T, Sellar CM, et al. (2009) Effects of presurgical exercise training on quality of life in patients undergoing lung resection for suspected malignancy: a pilot study. Cancer Nurs 32(2): 158-165.

30. Kendall F, Abreu P, Pinho P, Oliveira J, Bastos P, et al. (2017) The role of physiotherapy in patients undergoing pulmonary surgery for lung cancer. A literature review. Rev Port Pneumol (2006) 23(6): 343-351.

31. Charususin N, Gosselink R, Decramer M, McConnell A, Saey D, et 
al. (2013) Inspiratory muscle training protocol for patients with chronic obstructive pulmonary disease (IMTCO study): a multicentre randomised controlled trial. BMJ Open 3(8): e003101.

32. Lin PJ, Peppone LJ, Janelsins MC, Mohile SG, Kamen CS, et al. (2018) Yoga for the management of cancer treatment-related toxicities. Curr Oncol Rep 20(1): 5.

33. Kendrick KR, Baxi SC, Smith RM (2000) Usefulness of the modified 0-10 Borg scale in assessing the degree of dyspnea in patients with COPD and asthma. J Emerg Nurs 26(3): 216-222.

34. Kisner C, Colby LA (2007) Management of pulmonary conditions, in therapeutic exercise: Foundations and techniques. In: Davis FA (Ed.), Philedelphia, USA, pp. 851-882.
This work is licensed under Creative

Commons Attribution 4.0 License

DOI: 10.19080/JYP.2018.05.555664 